\title{
ESTIMACIÓN DEL CARBONO ALMACENADO EN EL BOSQUE NATURAL EN LA CUENCA MEDIA - BAJA. MUNICIPIO DE RÍO QUITO CHOCÓ, COLOMBIA
}

\author{
CARBON ESTIMATION STORAGED ON THE NATURAL FOREST ON THE MIDDLE - \\ LOWER, RIO QUITO TOWN, CHOCÓ, COLOMBIA
}

\author{
Cristian C Hurtado ${ }^{1}{ }^{*}$ Carlos H Corte, Max A Triana \\ Recibido para publicación: 18 de marzo 2017 - Aceptado para publicación: 29 de mayo 2017
}

\begin{abstract}
RESUMEN
En el ecosistema de bosque natural del Helobioma Pacífico -Atrato situado en la cuenca Media y Baja del municipio de Río Quito, se estimó la cantidad de biomasa aérea y carbono almacenado para el ecosistema y diez especies con mayor valor de importancia. Este ecosistema presenta precipitaciones anuales de 4000 a $8000 \mathrm{~mm}$. Los cálculos se realizaron con base en trece modelos alométricos que estiman biomasa en función del diámetro y altura total. Se utilizó un muestreo bietápico sobre 250 parcelas circulares de 0.05 ha. Los modelos seleccionados se basaron en los recomendados por el IPCC (2005); para la mayoría de las especies el modelo de mejor ajuste fue el de Chave et al. (2001). El cálculo del carbono almacenado se estimó con base en la fracción del $50 \%$ de la biomasa aérea. Los resultados obtenidos de biomasa y carbono fueron de 439.72 t/0.05 ha para biomasa y 219.86 t/0.05 ha para carbono almacenado siendo la especie de mayor aporte en biomasa Osteophloeum platyspermum con un 12.14\% del total del ecosistema y el carbono almacenado fue de $26.70 \mathrm{t} / 0.05$ ha. Las especies Osteophloeum platyspermum y Michropholis guyanensis presentan mayor número de individuos por hectárea.
\end{abstract}

Palabras clave: biomasa, carbono almacenado, clase diamétrica, modelos alométricos.

\begin{abstract}
On the ecosystem of the natural forest of Pacific Helobioma - Atrato, located on the middle lower basin of the Rio Quito town, it is estimated that the quantity of aerial biomass and carbon accumulated for the ecosystem and ten (10) species with the most important value. This ecosystem presents annual precipitations between 4000 to 8000 $\mathrm{mm}$. Calculations were made in basis in thirteen allometric models that estimate the biomass as a function of the diameter and the total height. A two-stage sampling over 250 round lots of 0.05 ha. The selected models were based on the ones that the IPCC recommended (2005); for most of the species, the model that adapted the most was the Chave et al. (2001). The calculation of the stocked carbon was estimated in basis of the fraction of the $50 \%$ of the aerial biomass. The results obtained of the biomass and carbon were of $439.72 \mathrm{t} / 0.05$ ha for biomass and 219.86 t/0.05 ha for stocked carbon, being the specie with more contribution on the biomass Osteophloeum platyspermum with a $12.14 \%$ of the total of the ecosystem and $26.70 \mathrm{t} / 0.05$ ha of the stocked carbon. The species Osteophloeum platyspermum and Michropholis guyanensis present the larger number of individuals per hectare.
\end{abstract}

Key words: Biomass, stocked carbon, diametric class, allomertric models.

\footnotetext{
${ }^{1}$ Estudiante pregrado en Ingeniería Forestal Universidad Distrital Francisco José de Caldas, Grupo semillero PROMAFOR UDFJC. Avenida calle 1 número 4 - 39. Bogotá D.C., Colombia

${ }^{2}$ Estudiante pregrado en Ingeniería Forestal Universidad Distrital Francisco José de Caldas, Grupo semillero PROMAFOR UDFJC. Calle 17c número 134-70, manzana J casa 3. Bogotá D.C., Colombia

${ }^{3}$ Magister en Manejo y Conservación de Recursos Naturales, Profesor, director del semillero PROMAFOR UDFJC, Especialista en manejo sostenible Universidad de la Amazonia, Ingeniero Forestal Universidad Distrital Francisco José de Caldas. Carrera 5 número 15-82, Bogotá, D. C., Colombia.

* Avenida Calle 1 número 4 - 39., Bogotá -Colombia, 3118341177, cchurtadov@correo.udistrital.edu.co
} 


\section{INTRODUCCIÓN}

Los bosques representan gran potencial como sumideros de carbono y por tanto son elementos clave a nivel del ecosistema para el balance y reducción de emisiones de CO2. La estimación de la capacidad de almacenamiento de Carbono que poseen estas formaciones vegetales constituye una labor fundamental para su valoración, conservación y uso sostenible. (Clark et al., 2001).

Colombia es un país con grandes riquezas naturales y extensas regiones con coberturas boscosas, sin embargo, existen aún vacíos de información asociados con su composición, estructura y diversidad, por tanto, las dificultades para estimar su capacidad de almacenamiento de carbono a escalas detalladas. A esta realidad se suma el peligro que representa su desaparición por cambio de uso del suelo, así para el año 2015, se identificaron a nivel nacional un total de 124.035 ha deforestadas de bosque natural, de las cuales el departamento del Chocó contribuyó con 5.813 ha lo que representa el $4,7 \%$ del total nacional para ese año. (Rangel 2006, IDEAM 2016).

Debido a estas problemáticas, existe la necesidad de generar datos sobre las reservas de carbono, los cuales son vitales para la evaluación, seguimiento y monitoreo tanto a nivel nacional como mundial. El objetivo de este trabajo es generar información que contribuya a estimar el contenido de carbono en la biomasa arbórea del ecosistema de bosque natural del helobioma Pacífico-Atrato en la cuenca baja y media del municipio de Río Quito en Chocó, para tal efecto se pretende resolver los siguientes interrogantes:

- ¿Cuánto representa la reserva de biomasa y de carbono en este ecosistema?.

- ¿Qué especies resultan ser las que mayor carbono almacenan?

- ¿Existen diferencias significativas para la estructura entre las especies de mayor índice de importancia, presentes en el ecosistema de bosques naturales del helobioma PacíficoAtrato?.

\section{MATERIALES Y MÉTODOS}

\section{1. Área de estudio}

El estudio se ubicó en la región del Pacífico Colombiano, en el departamento del Chocó, municipio de Río Quito, que se encuentra sobre el margen izquierdo aguas abajo del río Atrato. El municipio ocupa un área de 69.310 hectáreas, su cabecera municipal es Paimadó, pertenece a la cuenca hidrográfica del Río Atrato, se localiza geográficamente entre los $5^{\circ} 25^{\prime} 0^{\prime \prime}$ de Latitud Norte y entre los $76^{\circ} 40^{\prime} 00^{\prime \prime}$ de Longitud Oeste, los límites del municipio son por el norte con el municipio de Quibdó, por el occidente con el municipio de alto Baudó por el sur con el municipio de Cantón de San Pablo y por el oriente con el municipio de Atrato (Alcaldia Municipal Rio Quito, 2000).

\subsection{Características del área de estudio}

La altura del municipio varía desde los 0 hasta los 450 msnm, en cuanto a su clima, el área de estudio corresponde a la zona de vida de bosque muy húmedo tropical (bmh - T) según la clasificación de Holdridge, presenta precipitaciones de tipo monomodal entre los 4000 y 8000 mm anuales y una temperatura media mensual de $25^{\circ} \mathrm{C}$. Ocasionalmente se presentan fuertes vientos que causan la caída de árboles al interior del bosque provocando aperturas del dosel. La humedad relativa se mantiene arriba del $88 \%$, el ecosistema al que pertenece se encuentra dentro del gran bioma del bosque húmedo tropical, dividido a su vez por los ecosistemas Bosques naturales del helobioma Pacífico - Atrato, Vegetación secundaria del helobioma Pacífico - Atrato, Bosques naturales del zonobioma húmedo tropical del Pacífico - Atrato, vegetación secundaria del zonobioma húmedo tropical del Pacífico - Atrato y bosques naturales del orobioma de la serranía Baudó - Darién (IFCAYA 1975).

Según Villota (2005) la Ilanura del Pacífico y el valle del río Atrato, conforman una gran franja de origen aluvial, el área de estudio se sitúa hacia la parte media del río Atrato sobre su margen izquierda, conformada por las cuencas de los ríos Buey y Tagachí, en estas zonas se forman una serie de bateas o terrenos cóncavos que permanecen inundados desarrollando bosques de tipo guandal, creciendo en suelos pantanosos y muy sueltos desarrollando así muchas raíces, las formaciones geológicas se ubican cronológicamente en una secuencia que van desde el cretáceo hasta el cuaternario, mostrando así distintos paisajes que se han separado en la montaña. Se evidencian materiales del cretáceo en el piedemonte y lomerío materiales terciarios, en las planicies aluvial y fluviomarina y en los valles predominan sedimentos del cuaternario. 
Las pendientes del terreno varían de plano a muy escarpado, presenta siete unidades fisiográficas en las cuales se tienen basín, diques, complejos de orillares, terraza, valle intercolinar, colinas altas y colinas bajas (Ramirez 1967).

\subsection{Toma de datos}

El muestreo fue Bietápico por bloques y fajas con una intensidad de 0,08\%, y una probabilidad del $95 \%$, elaborado a través de cartografía obtenida del EOT del Municipio e imágenes satelitales (Landsat ETM+), donde se establecieron 250 parcelas circulares de 0,05 ha, distribuidas al azar en el área correspondiente al ecosistema de bosques naturales del helobioma Pacífico-Atrato, evaluando aspectos de composición y estructura de la vegetación según la metodología propuesta en (Rangel et al. 1997).

Para tal efecto se estableció un proceso de marcado de árboles que tuviesen un DAP superior a los $10 \mathrm{~cm}$, simultáneamente se realizó la captura y registro de datos con las variables DAP y altura, e identificación de especies por medio de recolecta de material vegetal para identificación taxonómica.

\subsection{Análisis de datos}

Posterior a este proceso se digitalizaron, filtraron y depuraron los datos registrados en las planillas de campo, como también cálculos de índices y estimación de modelos alométricos con ayuda de Excel 2010, para efectos valoración por especie se aplicó el índice de valor de importancia (IVI) (Acosta et al. 2006), con la fórmula:

$\mathrm{IVI}=\mathrm{Ar}+\mathrm{Dr}+\mathrm{Fr}$

Dónde:

IVI = Índice de valor de Importancia

$\mathrm{Ar}=$ Abundancia relativa

$\mathrm{Dr}=$ Dominancia relativa

$\mathrm{Fr}=$ Frecuencia relativa

Seleccionando las diez especies más importantes dentro del ecosistema, a quienes se les aplicaron trece modelos de estimación indirecta de biomasa expuestos en Alvarez $(2011,2012)$, Chave et al (2001, 2005), IPCC $(2005,2007)$ y Overman et al. (1990), descritos en la Tabla I para un total de 13 modelos evaluados, se realizó un modelo promedio para cada especie y el ecosistema en general, donde se seleccionó el modelo más cercano al modelo promedio a través de coeficientes de correlacion Spearman.

Tabla 1. Modelos alométricos utilizados en estimación de biomasa aérea para árboles con $D A P \geq 10.0 \mathrm{~cm}$ del bosque natural del Municipio de Rio Quito. Colombia.

\begin{tabular}{|c|c|}
\hline CÓDIGO DEL MODELO & MODELO \\
\hline Modelo1 (bh-MB) (Álvarez et al. 2011) & $\begin{aligned} B=\exp (2.226+ & (-1.552 * \ln D A P)+\left(1.237 * \ln D A P^{2}\right) \\
& \left.+\left(-0.126 * \ln D A P^{3}\right)+(-0.237 * \ln 0.65)\right)\end{aligned}$ \\
\hline Modelo2 (bh-PM) (Álvarez et al. 2011) & $\begin{aligned} B=\exp (2.421+ & (-1.415 * \ln D A P)+\left(1.237 * \ln D A P^{2}\right) \\
& \left.+\left(-0.126 * \ln D A P^{3}\right)+(1.068 * \ln 0.65)\right)\end{aligned}$ \\
\hline Modelo3 (bh-MB) (Álvarez et al. 2011) & $B=\exp (-1.663+(2.37 * \ln D A P))$ \\
\hline Modelo4 (bh-PM) (Álvarez et al. 2011) & $B=\exp (-1.866+(2.37 * \ln D A P))$ \\
\hline Modelo5 (bh-MB) (Álvarez et al. 2012) & $B=\exp \left(-1.993+\left(0.932 * \ln D A P^{2}\right) *(\right.$ altura $\left.m * 0.65)\right)$ \\
\hline Modelo6 (bh_PM) (Álvarez et al. 2012) & $B=\exp \left(-2.289+\left(0.932 * \ln D A P^{2}\right) *(\right.$ altura $\left.m * 0.65)\right)$ \\
\hline Modelo7 (bh-T) (Chave et al. 2001) & $B=\exp (-2.19+(2.54 * \ln D A P))$ \\
\hline Modelo8 (IPCC. 2005) & $B=\exp \left(2.289+(2.649 * \ln D A P)-\left(0.021 * \ln D A P^{2}\right)\right)$ \\
\hline Modelo9 (Chave et al.. 2005) & $\begin{array}{c}B=0.65 \exp \left(-1.499+(2.148 * \ln D A P)+\left(0.207 * \ln D A P^{2}\right)\right. \\
\left.-\left(0.0281 * \ln D A P^{3}\right)\right)\end{array}$ \\
\hline Modelo10 (Chave et al.. 2005) & $\begin{array}{c}B=0.65 \exp \left(-0.667+(1.784 * \ln D A P)+\left(0.207 * \ln D A P^{2}\right)\right. \\
\left.-\left(0.0281 * \ln D A P^{3}\right)\right)\end{array}$ \\
\hline Modelo11 (Overman et al. 1990) & $B=\exp \left(-2.904+\left(0.993 * \ln \left(\pi * D A P^{2} *\right.\right.\right.$ altura $\left.\left.\left.m\right)\right)\right)$ \\
\hline Modelo12 (Chave et al. 2005) & $B=\exp \left(-2.977+\left(\ln \left(0.65 * D A P^{2} *\right.\right.\right.$ altura $\left.\left.\left.m\right)\right)\right)$ \\
\hline Modelo13 (Chave et al. 2005) & $B=\exp \left(-2.187+\left(0.916 * \ln \left(0.65 * D A P^{2} *\right.\right.\right.$ altura $\left.\left.\left.m\right)\right)\right)$ \\
\hline
\end{tabular}


Una vez procesados estos datos fueron ingresados al software libre $R$ Project 3.1.1 estableciendo el análisis estadístico descriptivo (salidas gráficas, media, desviación estándar e intervalos de confianza, normalidad de datos) e inferencial (pruebas de varianza), el carbono almacenado se calculó de acuerdo con el IPCC (2011) como el 50\% de la biomasa estimada de cada modelo seleccionado, tanto para especie como para ecosistema en general. Todas las estimaciones se realizaron a 0.05 ha con el fin de evitar la propagación de errores con respecto a las sobreestimaciones a que conllevan los modelos alométricos (Arellano \& Vásquez, 2012).

\section{ANÁLISIS Y DISCUSIÓN}

\subsection{Selección de especies}

La selección de las especies más importantes dentro del ecosistema con base al IVI, se presentan en la Tabla II, donde las familias Mimosaceae y Myristicaceae son las que más frecuentes, y la especie con mayor IVI es E. coriácea, con un valor de $5.37 \%$, y la décima especie seleccionada es $A$. aspera con un valor de $2.53 \%$.

\subsection{Estimación de biomasa}

Las estimaciones de los modelos con mayor coeficiente de correlación de Spearman presentaron valores idénticos, debido a esto la selección del modelo más ajustado se realizó por medio de la localización más idónea con respecto a la zona donde el autor recomienda que se utilice. Para todas las especies y ecosistema general, exceptuando E. coriácea y Aspidosperma sp. (cuyo modelo fue siete y diez respectivamente) el modelo seleccionado fue el nueve Tabla I.

Tabla 2. Valores de cálculo de IVI y valores medios de estructura. biomasa y carbono. contrastados mediante la prueba (Kruskal-Wallis).

\begin{tabular}{|c|c|c|c|c|c|c|}
\hline Especie & IVI & $\begin{array}{l}\text { Densidad } \\
\text { (ind/0.05 } \\
\text { ha) }\end{array}$ & $\begin{array}{l}\text { Altura } \\
\text { total (m) }\end{array}$ & $\begin{array}{l}\text { Área basal } \\
\text { (m2/0.05 ha) }\end{array}$ & $\begin{array}{l}\text { Biomasa } \\
\text { (t/0.05 ha) }\end{array}$ & $\begin{array}{l}\text { Carbono } \\
\text { (t/0.05 ha) }\end{array}$ \\
\hline $\begin{array}{l}\text { Eschweilera coriacea (DC.) S.A. } \\
\text { Mori }\end{array}$ & 5.37 & 24.08 & 11.34 & 1.54 & $\begin{array}{l}87.68+/- \\
0.40\end{array}$ & 43.80 \\
\hline Manicaria saccifera Gaertn. & 5.25 & 38.42 & 5.88 & 1.49 & $\begin{array}{l}40.64+/- \\
0.26\end{array}$ & 20.32 \\
\hline $\begin{array}{l}\text { Michropholis guyanensis (A. DC.) } \\
\text { Pierre }\end{array}$ & 4.39 & 23.53 & 12.98 & 1.88 & $\begin{array}{l}45.08+/- \\
0.79\end{array}$ & 22.54 \\
\hline Iryanthera juruensis Warb. & 4.03 & 23.83 & 11.02 & 0.88 & $\begin{array}{l}29.63+/- \\
0.20\end{array}$ & 14.82 \\
\hline $\begin{array}{l}\text { Osteophloeum platyspermum } \\
\text { (Spruce ex A.DC.) Warb. }\end{array}$ & 3.34 & 23.08 & 18.87 & 4.87 & $\begin{array}{l}53.39+/- \\
2.96\end{array}$ & 26.70 \\
\hline Aspidosperma sp. & 3.31 & 23.75 & 15.32 & 3.37 & $\begin{array}{c}43.38+/- \\
1.30\end{array}$ & 21.69 \\
\hline $\begin{array}{l}\text { Pentaclethra macroloba (Willd.) } \\
\text { Kuntze }\end{array}$ & 2.92 & 24.21 & 12.57 & 2.08 & $\begin{array}{l}29.94+/- \\
0.74\end{array}$ & 14.97 \\
\hline Inga acrocephala Steud. & 2.80 & 24.24 & 11.35 & 0.60 & $\begin{array}{l}14.58+/- \\
0.26\end{array}$ & 7.29 \\
\hline Apeiba aspera Aubl. & 2.53 & 20.00 & 11.70 & 1.35 & $\begin{array}{l}23.93+/- \\
0.71\end{array}$ & 11.97 \\
\hline Guarea aff. glabra Vahl & 2.68 & 20.00 & 15.42 & 2.04 & $\begin{array}{l}29.57+/- \\
0.75 \\
\end{array}$ & 14.79 \\
\hline \multicolumn{2}{|c|}{$\begin{array}{c}\text { Ecosistema de Bosques Naturales del } \\
\text { Heliobioma Pacifico-Atrato }\end{array}$} & 78.55 & 11.45 & 3.70 & $\begin{array}{l}32.26+/- \\
0.27\end{array}$ & 16.13 \\
\hline \multicolumn{2}{|l|}{ Estadístico H (10. N=367) } & 235.90 & 137.86 & 36.57 & \multicolumn{2}{|c|}{36.57} \\
\hline \multicolumn{2}{|l|}{ Nivel de significancia $(p)$} & $<2.2 \mathrm{e}-16$ & $<2.2 \mathrm{e}-16$ & 0.00 & \multicolumn{2}{|c|}{0.00} \\
\hline
\end{tabular}


La distribución de biomasa se muestra en la Figura I, con respecto a cada clase diamétrica para los trece modelos utilizados tanto a nivel de ecosistema como para las especies seleccionadas. Se observa una divergencia en la estimación, ya que los modelos 8 y 2 proponen cantidades de biomasa mayores que los modelos 13 y 12, siendo concordante con la literatura revisada.
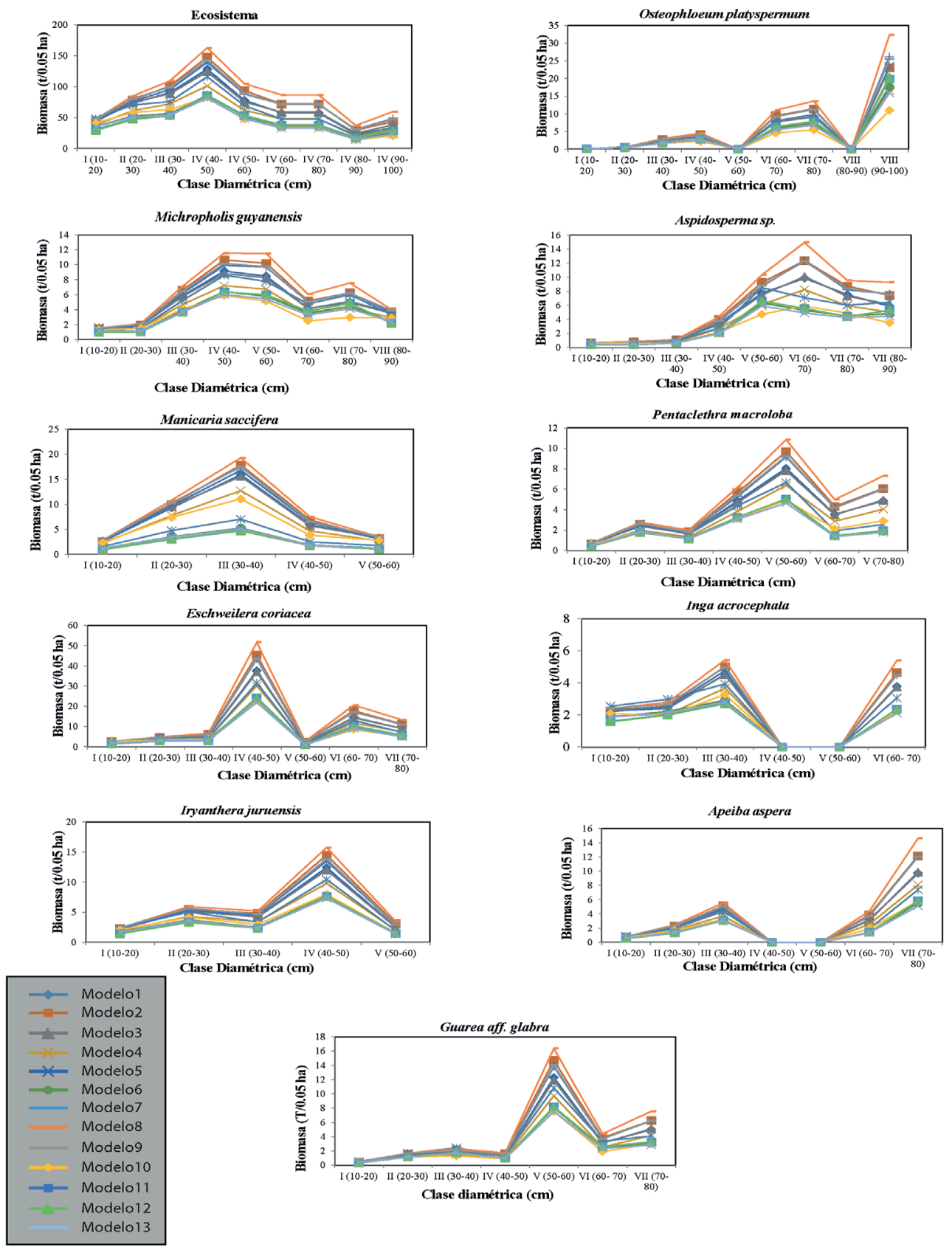

Se presento una marcada tendencia en los modelos que estiman la biomasa en función del DAP ( 8,2 y 9) quienes estimaron mayor cantidad de biomasa con respecto a los modelos calculados en función de DAP y altura total (12 y 13) quienes estimaron menor cantidad de biomasa, esto es constante tanto en cálculos para el ecosistema como para especies seleccionadas, tal como puede verse en la Figura II.
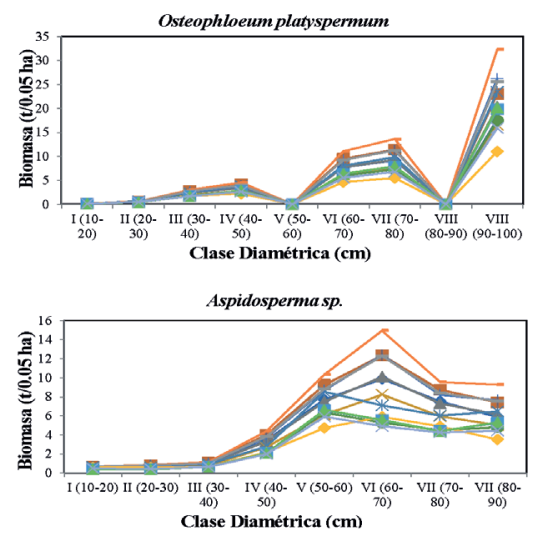

Clase diamétrica $(\mathrm{cm})$

Figura 1. Distribución de biomasa aérea por clase diamétrica $(\mathrm{cm})$ usando trece modelos alométricos Tabla I, basados en literatura. 

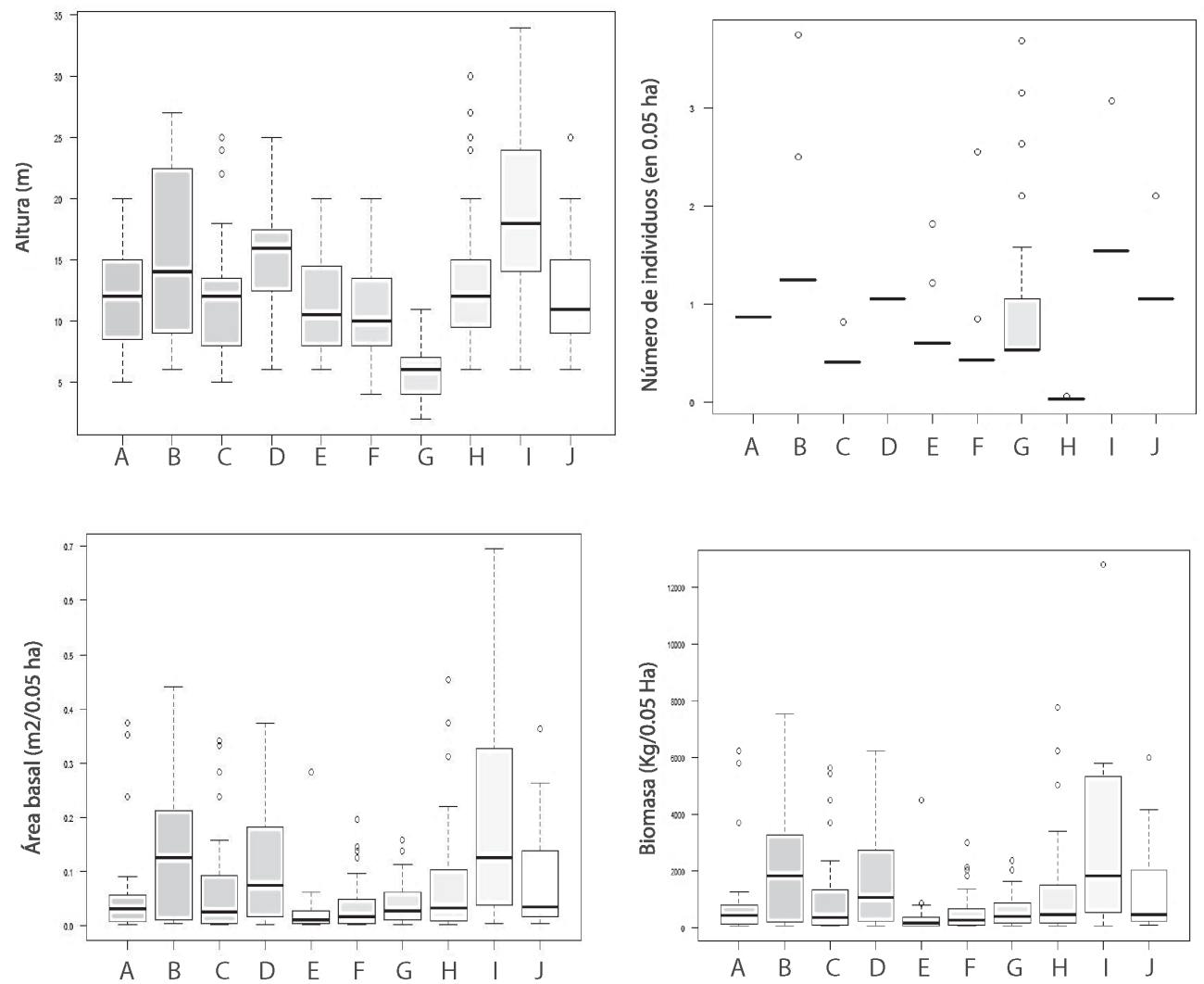

Figura 2. Variación de parámetros de estructura (Densidad, altura, área basal) y biomasa para las especies: A. Apeiba aspera, B. Aspidosperma sp, C. Eschweilera coriacea, D. Guarea a. glabra, E. Inga acrocephala, F. Iryanthera juruensis, G. Manicaria saccifera, H. Michropholis guyanensis, I. Osteophloeum platyspermum, J. Pentaclethra macroloba.

\subsection{Estructura y biomasa}

Con el fin de establecer la existencia de diferencias significativas entre las variables estructurales tanto de ecosistema como de las especies seleccionadas se aplicó la prueba no paramétrica equivalente para análisis de varianza de Kruskalwallis, donde todas las variables (densidad, alturas, biomasa), presentaron diferencias significativas (con una probabilidad del $0.05 \%$ ), tanto a nivel de ecosistema como para cada una de las especies seleccionadas. Estas variaciones de estructura y biomasa se pueden observar de manera clara en la Tabla II.

Los valores promedio más altos de área basal se presentan en la especie 0 . platyspermum con $4.87 \mathrm{~m}^{2} / 0.05$ ha, y la más baja de $0.60 \mathrm{~m}^{2} / 0.05$ ha para (I. acrocephala), para la densidad promedio en 0.05 ha se obtuvo el mayor valor para $(M$. saccifera) con 38.42, mientras que la menor para las especies ( $A$. aspera y $G$. aff. glabra), con valores medios de 20 individuos. A nivel de ecosistema la densidad media fue de 78,55 ind. /0.05 ha, ver Tabla II.

La especie con mediana más alta en altura con respecto a las demás es ( $O$. platyspermum) con valor de $18 \mathrm{~m}$, mientras que la más baja tiene $6 \mathrm{~m}$ para la especie (M. saccifera), la mayor dispersión de área basal es (O. platyspermum), y con menor dispersión (I. acrocephala), tal y como se observa en los boxplot generados en la Figura II.

Distribución de variabilidad estructural y de biomasa por clase diamétrica.

En conjunto las alturas de las especies más representativas del ecosistema presentan una variación de 33 metros, que van desde los 3 metros de altura hasta los 34, mientras que para las especies particulares la mayor variación de altura es para M. guyanensis y O. platyspermum, 
con más de 24 metros entre su altura más baja y más alta. Algunas especies presentan ausencias en alguna de sus clases diamétricas ( $I$. juruensis y M. saccifera), situación muy común en este tipo de bosques, sujetos a perturbaciones de baja y mediana intensidad.

La distribución relativa de biomasa calculada para el ecosistema y para la especie 0 . platyspermum muestra como es natural en bosques de baja a media intervención, una gran acumulación hacia las últimas clases (IX 90-100). Sin embrago para especies puntuales como $M$. saccifera, I. acrocephala, la clase diamétrica con mayor biomasa es la clase III (30-40), y de igual forma para las especies $P$. macroloba y G. aff. Glabra sobresale la clase V (50-60), por último, la especie Aspidosperma presenta acumulaciones importantes en torno a la clase VI (60-70), característica que aduce a una condición discetánea del bosque Figura III y Tabla III.
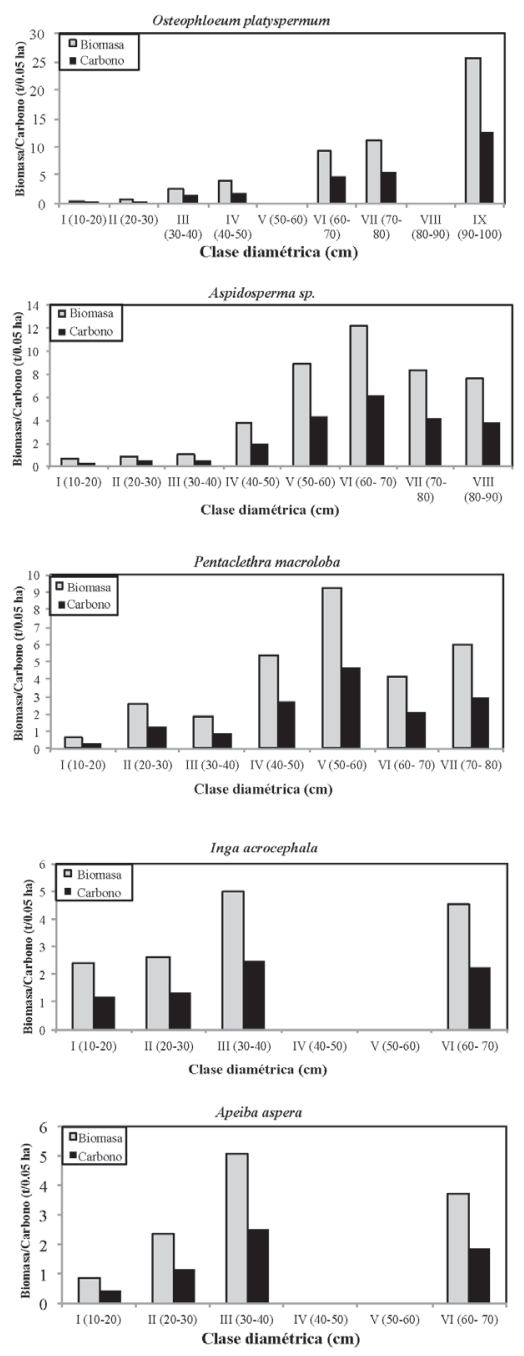

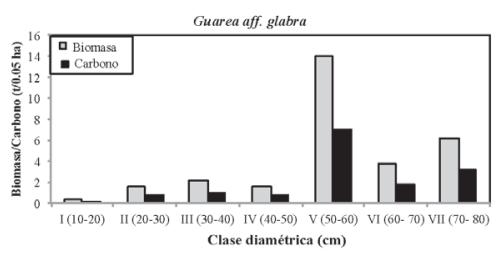

Figura 3. Distribución de la biomasa aérea y carbono por clase diamétrica de los individuos con DAP>10 cm de las diferentes especies del ecosistema. 
Tabla 3. Distribución de las variables de la estructura de la vegetación. biomasa y carbono por clase diamétrica para las especies seleccionadas y el ecosistema.

\begin{tabular}{|c|c|c|c|c|c|c|c|c|c|c|c|}
\hline \multirow{7}{*}{ 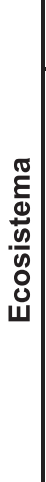 } & Variable & $\begin{array}{l}\mathrm{I}(10- \\
20)\end{array}$ & $\begin{array}{c}\text { II (20- } \\
30)\end{array}$ & $\begin{array}{c}\text { III (30- } \\
40)\end{array}$ & $\begin{array}{c}\text { IV (40- } \\
50)\end{array}$ & $\begin{array}{c}\text { V (50- } \\
60) \\
\end{array}$ & $\begin{array}{c}\text { VI (60- } \\
70)\end{array}$ & $\begin{array}{c}\text { VII (70- } \\
80) \\
\end{array}$ & $\begin{array}{l}\text { VIII (80- } \\
90) \\
\end{array}$ & $\begin{array}{c}\text { IX (90- } \\
100) \\
\end{array}$ & Total \\
\hline & Altura (m) & $\begin{array}{l}3.0- \\
25.0\end{array}$ & $\begin{array}{l}2.2- \\
30.0\end{array}$ & $\begin{array}{l}4.0- \\
31.0\end{array}$ & $\begin{array}{l}5.0- \\
29.0\end{array}$ & $\begin{array}{l}4.0- \\
26.0\end{array}$ & $\begin{array}{l}11.0- \\
26.0\end{array}$ & $\begin{array}{l}11.0- \\
30.0\end{array}$ & $\begin{array}{l}15.0- \\
25.0\end{array}$ & $\begin{array}{l}13.0- \\
34.0\end{array}$ & $\begin{array}{l}3.0- \\
34.0\end{array}$ \\
\hline & Densidad & 46.73 & 30.00 & 28.00 & 24.29 & 21.43 & 13.57 & 21.82 & 20.00 & 20.00 & 25.09 \\
\hline & $\begin{array}{l}\text { Área basal } \\
\text { (m2/0.05ha) }\end{array}$ & 0.29 & 0.82 & 1.81 & 2.85 & 4.21 & 3.69 & 7.85 & 8.90 & 13.03 & 43.44 \\
\hline & $\begin{array}{l}\text { Biomasa } \\
\text { (t/0.05ha) }\end{array}$ & 2.86 & 6.53 & 13.42 & 24.52 & 37.90 & 56.26 & 68.47 & 86.71 & 143.06 & 439.72 \\
\hline & $\begin{array}{c}\text { Biomasa relativa } \\
(\%)\end{array}$ & 0.65 & 1.48 & 3.05 & 5.58 & 8.62 & 12.79 & 15.57 & 19.72 & 32.53 & 100.00 \\
\hline & $\begin{array}{c}\text { Carbono (t/0.05 } \\
\text { ha) }\end{array}$ & 1.43 & 3.26 & 6.71 & 12.26 & 18.95 & 28.13 & 34.23 & 43.36 & 71.53 & 219.86 \\
\hline \multirow{6}{*}{ 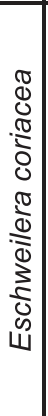 } & Altura (m) & $\begin{array}{l}5.0- \\
16.0\end{array}$ & $\begin{array}{l}7.0- \\
25.0\end{array}$ & $\begin{array}{l}9.0- \\
16.0\end{array}$ & $\begin{array}{l}13.0- \\
18.0\end{array}$ & 12.00 & $\begin{array}{l}15.0- \\
24.0\end{array}$ & $\begin{array}{l}12.0- \\
22.0\end{array}$ & 0.00 & 0.00 & $\begin{array}{l}5.0- \\
22.0\end{array}$ \\
\hline & Densidad & 21.54 & 22.22 & 20.00 & 26.60 & 20.00 & 20.00 & 20.00 & 0.00 & 0.00 & 16.71 \\
\hline & $\begin{array}{l}\text { Área basal } \\
\text { (m2/0.05ha) }\end{array}$ & 2.70 & 4.63 & 5.97 & 43.81 & 2.35 & 17.21 & 11.02 & 0.00 & 0.00 & 87.69 \\
\hline & $\begin{array}{l}\text { Biomasa } \\
\text { (t/0.05ha) }\end{array}$ & 0.10 & 0.76 & 1.44 & 2.31 & 2.35 & 3.18 & 6.74 & 0.00 & 0.00 & 16.89 \\
\hline & $\begin{array}{c}\text { Biomasa relativa } \\
(\%)\end{array}$ & 3.08 & 5.28 & 6.80 & 49.96 & 2.68 & 6.19 & 12.57 & 0.00 & 0.00 & 100.00 \\
\hline & $\begin{array}{c}\text { Carbono (t/0.05 } \\
\text { ha) }\end{array}$ & 0.05 & 0.38 & 0.72 & 1.15 & 1.18 & 1.59 & 3.37 & 0.00 & 0.00 & 8.44 \\
\hline \multirow{6}{*}{ 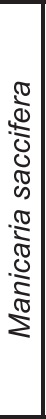 } & Altura (m) & $\begin{array}{l}2.0- \\
11.0\end{array}$ & $2.2-9.0$ & $\begin{array}{l}6.0- \\
14.0\end{array}$ & $5.0-8.0$ & 4.00 & 0.00 & 0.00 & 0.00 & 0.00 & $\begin{array}{l}2.0- \\
14.0\end{array}$ \\
\hline & Densidad & 40.00 & 30.00 & 29.23 & 20.00 & 20.00 & 0.00 & 0.00 & 0.00 & 0.00 & 15.47 \\
\hline & $\begin{array}{l}\text { Área basal } \\
\text { (m2/0.05ha) }\end{array}$ & 0.34 & 0.85 & 1.96 & 2.31 & 3.18 & 0.00 & 0.00 & 0.00 & 0.00 & 8.63 \\
\hline & $\begin{array}{l}\text { Biomasa } \\
\text { (t/0.05ha) }\end{array}$ & 2.81 & 10.28 & 17.49 & 6.60 & 3.46 & 0.00 & 0.00 & 0.00 & 0.00 & 40.64 \\
\hline & $\begin{array}{c}\text { Biomasa relativa } \\
(\%)\end{array}$ & 6.92 & 25.29 & 43.04 & 16.24 & 8.50 & 0.00 & 0.00 & 0.00 & 0.00 & 100.00 \\
\hline & $\begin{array}{c}\text { Carbono (t/0.05 } \\
\text { ha) }\end{array}$ & 0.17 & 0.42 & 0.98 & 1.15 & 1.59 & 0.00 & 0.00 & 0.00 & 0.00 & 20.32 \\
\hline \multirow{6}{*}{ 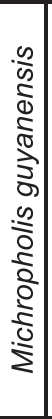 } & Altura (m) & $\begin{array}{l}6.0- \\
15.0\end{array}$ & $\begin{array}{l}6.0- \\
12.0\end{array}$ & $\begin{array}{l}12.0- \\
16.0\end{array}$ & $\begin{array}{l}7.0- \\
30.0\end{array}$ & $\begin{array}{l}12.0- \\
24.0\end{array}$ & 25.00 & 27.00 & 15.00 & 0.00 & $\begin{array}{l}6.0- \\
30.0\end{array}$ \\
\hline & Densidad & 23.08 & 20.00 & 20.00 & 20.00 & 20.00 & 20.00 & 20.00 & 20.00 & 0.00 & 18.12 \\
\hline & $\begin{array}{l}\text { Área basal } \\
\text { (m2/0.05ha) }\end{array}$ & 0.15 & 0.50 & 1.34 & 2.36 & 4.25 & 6.23 & 7.48 & 9.07 & 0.00 & 31.39 \\
\hline & $\begin{array}{l}\text { Biomasa } \\
\text { (t/0.05ha) }\end{array}$ & 1.54 & 2.03 & 6.45 & 10.18 & 9.77 & 5.04 & 6.21 & 3.86 & 0.00 & 45.09 \\
\hline & $\begin{array}{c}\text { Biomasa relativa } \\
(\%)\end{array}$ & 3.43 & 4.51 & 14.31 & 22.57 & 21.66 & 11.17 & 13.78 & 8.57 & 0.00 & 100.00 \\
\hline & $\begin{array}{c}\text { Carbono (t/0.05 } \\
\text { ha) }\end{array}$ & 0.77 & 1.02 & 3.23 & 5.09 & 4.88 & 2.52 & 3.11 & 1.93 & 0.00 & 22.54 \\
\hline \multirow{6}{*}{ 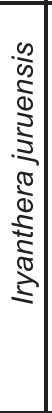 } & Altura (m) & $\begin{array}{l}7.0- \\
15.0\end{array}$ & $\begin{array}{l}8.0- \\
20.0\end{array}$ & $\begin{array}{l}9.0- \\
17.0\end{array}$ & $\begin{array}{l}11.0- \\
18.0\end{array}$ & 15.00 & 0.00 & 0.00 & 0.00 & 0.00 & $\begin{array}{l}7.0= \\
20.0\end{array}$ \\
\hline & Densidad & 22.73 & 21.25 & 20.00 & 20.00 & 20.00 & 0.00 & 0.00 & 0.00 & 0.00 & 11.55 \\
\hline & $\begin{array}{l}\text { Área basal } \\
\text { (m2/0.05ha) }\end{array}$ & 0.14 & 0.52 & 1.37 & 2.41 & 3.93 & 0.00 & 0.00 & 0.00 & 0.00 & 8.37 \\
\hline & $\begin{array}{l}\text { Biomasa } \\
\text { (t/0.05ha) }\end{array}$ & 2.43 & 5.67 & 4.72 & 13.84 & 2.98 & 0.00 & 0.00 & 0.00 & 0.00 & 29.64 \\
\hline & $\begin{array}{c}\text { Biomasa relativa } \\
(\%)\end{array}$ & 8.21 & 19.12 & 15.91 & 46.71 & 10.05 & 0.00 & 0.00 & 0.00 & 0.00 & 100.00 \\
\hline & $\begin{array}{c}\text { Carbono (t/0.05 } \\
\text { ha) }\end{array}$ & 1.22 & 2.83 & 2.36 & 6.92 & 1.49 & 0.00 & 0.00 & 0.00 & 0.00 & 14.82 \\
\hline
\end{tabular}




\begin{tabular}{|c|c|c|c|c|c|c|c|c|c|c|c|}
\hline \multirow{7}{*}{ 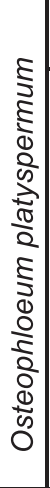 } & Variable & $\begin{array}{l}I(10- \\
20)\end{array}$ & $\begin{array}{l}\text { II (20- } \\
30)\end{array}$ & $\begin{array}{l}\text { III (30- } \\
40)\end{array}$ & $\begin{array}{c}\text { IV (40- } \\
50)\end{array}$ & $\begin{array}{l}V(50- \\
60)\end{array}$ & $\begin{array}{l}\text { VI (60- } \\
70)\end{array}$ & $\begin{array}{l}\text { VII (70- } \\
80)\end{array}$ & $\begin{array}{l}\text { VIII (80- } \\
90)\end{array}$ & $\begin{array}{l}\text { IX (90- } \\
100)\end{array}$ & Total \\
\hline & Altura (m) & $\begin{array}{l}6.0- \\
10.0\end{array}$ & $\begin{array}{l}12.0- \\
18.0\end{array}$ & $\begin{array}{l}13.0- \\
16.0\end{array}$ & $\begin{array}{l}18.0- \\
22.0\end{array}$ & 0.00 & $\begin{array}{l}18.0- \\
26.0\end{array}$ & $\begin{array}{l}17.0- \\
30.0\end{array}$ & 0.00 & $\begin{array}{l}28.0- \\
24.0\end{array}$ & $\begin{array}{l}6.0- \\
30.0\end{array}$ \\
\hline & Densidad & 20.00 & 20.00 & 20.00 & 13.33 & 0.00 & 13.33 & 13.33 & 0.00 & 13.33 & 12.59 \\
\hline & $\begin{array}{l}\text { Área basal } \\
\text { (m2/0.05ha) }\end{array}$ & 0.10 & 0.40 & 1.33 & 1.81 & 0.00 & 3.85 & 4.56 & 0.00 & 9.25 & 21.30 \\
\hline & $\begin{array}{l}\text { Biomasa } \\
\text { (t/0.05ha) }\end{array}$ & 0.17 & 0.55 & 2.73 & 3.94 & 0.00 & 9.23 & 11.22 & 0.00 & 25.56 & 53.40 \\
\hline & $\begin{array}{c}\text { Biomasa relativa } \\
(\%)\end{array}$ & 0.32 & 1.03 & 5.12 & 7.37 & 0.00 & 17.29 & 21.01 & 0.00 & 47.86 & 100.00 \\
\hline & $\begin{array}{c}\text { Carbono (t/0.05 } \\
\text { ha) }\end{array}$ & 0.05 & 0.20 & 0.66 & 0.90 & 0.00 & 1.92 & 2.28 & 0.00 & 4.63 & 26.70 \\
\hline \multirow{6}{*}{$\begin{array}{l}0 \\
5 \\
0 \\
\frac{1}{0} \\
\frac{1}{0} \\
0 \\
\frac{0}{0} \\
\frac{2}{4}\end{array}$} & Altura (m) & $\begin{array}{l}8.0- \\
11.0\end{array}$ & $\begin{array}{l}9.0- \\
10.0\end{array}$ & 15.00 & $\begin{array}{l}9.0- \\
20.0\end{array}$ & $\begin{array}{l}14.0- \\
27.0\end{array}$ & 26.00 & $\begin{array}{l}15.0- \\
16.0\end{array}$ & 25.00 & 0.00 & $\begin{array}{l}8.0- \\
27.0\end{array}$ \\
\hline & Densidad & 20.00 & 20.00 & 20.00 & 40.00 & 26.67 & 20.00 & 40.00 & 20.00 & 0.00 & 22.96 \\
\hline & $\begin{array}{l}\text { Área basal } \\
\text { (m2/0.05ha) }\end{array}$ & 0.15 & 0.60 & 5.28 & 2.64 & 5.35 & 5.47 & 14.78 & 8.84 & 0.00 & 43.11 \\
\hline & $\begin{array}{l}\text { Biomasa } \\
\text { (t/0.05ha) }\end{array}$ & 0.69 & 0.81 & 1.04 & 3.83 & 8.81 & 12.27 & 8.40 & 7.54 & 0.00 & 43.38 \\
\hline & $\begin{array}{c}\text { Biomasa relativa } \\
(\%)\end{array}$ & 1.58 & 1.86 & 2.40 & 8.83 & 20.31 & 28.28 & 19.36 & 17.37 & 0.00 & 100.00 \\
\hline & $\begin{array}{c}\text { Carbono (t/0.05 } \\
\text { ha) }\end{array}$ & 0.34 & 0.40 & 0.52 & 1.91 & 4.41 & 6.13 & 4.20 & 3.77 & 0.00 & 21.69 \\
\hline \multirow{6}{*}{ 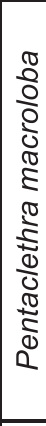 } & Altura (m) & $\begin{array}{l}9.0- \\
14.0\end{array}$ & $\begin{array}{l}6.0- \\
20.0\end{array}$ & $\begin{array}{l}9.0- \\
20.0\end{array}$ & $\begin{array}{l}7.0- \\
25.0\end{array}$ & $\begin{array}{l}14.0- \\
19.0\end{array}$ & 11.00 & 11.00 & 0.00 & 0.00 & $\begin{array}{l}6.0= \\
25.0\end{array}$ \\
\hline & Densidad & 20.00 & 23.33 & 6.67 & 20.00 & 20.00 & 20.00 & 20.00 & 0.00 & 0.00 & 14.44 \\
\hline & $\begin{array}{l}\text { Área basal } \\
\text { (m2/0.05ha) }\end{array}$ & 0.15 & 0.65 & 0.44 & 2.49 & 4.04 & 5.28 & 7.26 & 0.00 & 0.00 & 20.32 \\
\hline & $\begin{array}{l}\text { Biomasa } \\
\text { (t/0.05ha) }\end{array}$ & 0.70 & 2.62 & 1.81 & 5.40 & 9.24 & 4.17 & 6.01 & 0.00 & 0.00 & 29.95 \\
\hline & $\begin{array}{c}\text { Biomasa relativa } \\
(\%)\end{array}$ & 2.33 & 8.75 & 6.05 & 18.04 & 30.85 & 13.92 & 20.06 & 0.00 & 0.00 & 100.00 \\
\hline & $\begin{array}{c}\text { Carbono (t/0.05 } \\
\text { ha) }\end{array}$ & 0.35 & 1.31 & 0.91 & 2.70 & 4.62 & 2.08 & 3.00 & 0.00 & 0.00 & 14.97 \\
\hline \multirow{6}{*}{ 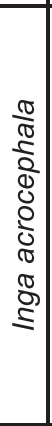 } & Altura (m) & $\begin{array}{l}6.0- \\
18.0\end{array}$ & $\begin{array}{l}8.0- \\
20.0\end{array}$ & $\begin{array}{l}10.0- \\
18.0\end{array}$ & 0.00 & 0.00 & 17.00 & 0.00 & 0.00 & 0.00 & $\begin{array}{l}6.0- \\
20.0\end{array}$ \\
\hline & Densidad & 22.86 & 22.86 & 20.00 & 0.00 & 0.00 & 20.00 & 0.00 & 0.00 & 0.00 & 9.52 \\
\hline & $\begin{array}{l}\text { Área basal } \\
\text { (m2/0.05ha) }\end{array}$ & 0.15 & 0.56 & 1.05 & 0.00 & 0.00 & 5.65 & 0.00 & 0.00 & 0.00 & 7.42 \\
\hline & $\begin{array}{l}\text { Biomasa } \\
\text { (t/0.05ha) }\end{array}$ & 2.43 & 2.66 & 4.99 & 0.00 & 0.00 & 4.51 & 0.00 & 0.00 & 0.00 & 14.59 \\
\hline & $\begin{array}{c}\text { Biomasa relativa } \\
(\%)\end{array}$ & 16.63 & 18.26 & 34.22 & 0.00 & 0.00 & 30.89 & 0.00 & 0.00 & 0.00 & 100.00 \\
\hline & $\begin{array}{c}\text { Carbono (t/0.05 } \\
\text { ha) }\end{array}$ & 1.21 & 1.33 & 2.50 & 0.00 & 0.00 & 2.25 & 0.00 & 0.00 & 0.00 & 7.29 \\
\hline \multirow{6}{*}{$\begin{array}{l}\frac{\pi}{0} \\
\frac{2}{2} \\
\frac{\pi}{\pi} \\
\frac{\pi}{0} \\
\frac{2}{0}\end{array}$} & Altura (m) & $\begin{array}{l}5.0- \\
14.0\end{array}$ & $\begin{array}{l}6.0- \\
15.0\end{array}$ & $\begin{array}{l}10.0- \\
20.0\end{array}$ & 0.00 & 0.00 & 12.00 & $\begin{array}{l}16.0- \\
17.0\end{array}$ & 0.00 & 0.00 & $\begin{array}{l}5.0- \\
20.0\end{array}$ \\
\hline & Densidad & 20.00 & 20.00 & 20.00 & 0.00 & 0.00 & 20.00 & 40.00 & 0.00 & 0.00 & 13.33 \\
\hline & $\begin{array}{l}\text { Área basal } \\
\text { (m2/0.05ha) }\end{array}$ & 0.13 & 0.58 & 1.23 & 0.00 & 0.00 & 0.79 & 7.26 & 0.00 & 0.00 & 10.00 \\
\hline & $\begin{array}{l}\text { Biomasa } \\
\text { (t/0.05ha) }\end{array}$ & 0.83 & 2.34 & 5.05 & 0.00 & 0.00 & 3.70 & 12.02 & 0.00 & 0.00 & 23.93 \\
\hline & $\begin{array}{c}\text { Biomasa relativa } \\
(\%)\end{array}$ & 3.47 & 9.76 & 21.11 & 0.00 & 0.00 & 15.44 & 50.21 & 0.00 & 0.00 & 100.00 \\
\hline & $\begin{array}{c}\text { Carbono (t/0.05 } \\
\text { ha) }\end{array}$ & 0.42 & 1.17 & 2.53 & 0.00 & 0.00 & 1.85 & 6.01 & 0.00 & 0.00 & 11.97 \\
\hline
\end{tabular}




\begin{tabular}{|c|c|c|c|c|c|c|c|c|c|c|c|}
\hline \multirow{7}{*}{ 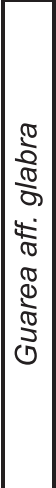 } & Variable & $\begin{array}{r}I(10- \\
20)\end{array}$ & $\begin{array}{c}11(20- \\
30)\end{array}$ & $\begin{array}{l}\text { III (30- } \\
40)\end{array}$ & $\begin{array}{c}\text { IV (40- } \\
50)\end{array}$ & $\begin{array}{l}\mathbf{V}(50- \\
60)\end{array}$ & $\begin{array}{l}\text { VI (60- } \\
70)\end{array}$ & $\begin{array}{l}\text { VII (70- } \\
80)\end{array}$ & $\begin{array}{l}\text { VIII (80- } \\
90)\end{array}$ & $\begin{array}{c}\text { IX (90- } \\
100) \\
\end{array}$ & Total \\
\hline & Altura (m) & $\begin{array}{l}6.0- \\
16.0\end{array}$ & $\begin{array}{l}12.0- \\
16.0\end{array}$ & $\begin{array}{l}16.0- \\
25.0\end{array}$ & 17.00 & $\begin{array}{l}14.0- \\
20.0\end{array}$ & 22.00 & 18.00 & 0.00 & 0.00 & $\begin{array}{l}6.0- \\
25.0\end{array}$ \\
\hline & Densidad & 20.00 & 20.00 & 20.00 & 20.00 & 20.00 & 20.00 & 20.00 & 0.00 & 0.00 & 15.56 \\
\hline & $\begin{array}{l}\text { Área basal } \\
\text { (m2/0.05ha) }\end{array}$ & 0.10 & 0.60 & 1.51 & 2.15 & 18.59 & 4.75 & 7.48 & 0.00 & 0.00 & 35.18 \\
\hline & $\begin{array}{l}\text { Biomasa } \\
\text { (t/0.05ha) }\end{array}$ & 0.43 & 1.62 & 2.08 & 1.53 & 14.01 & 3.70 & 6.21 & 0.00 & 0.00 & 29.58 \\
\hline & $\begin{array}{c}\text { Biomasa relativa } \\
(\%)\end{array}$ & 1.44 & 5.47 & 7.05 & 5.16 & 47.38 & 12.49 & 21.01 & 0.00 & 0.00 & 100.00 \\
\hline & $\begin{array}{c}\text { Carbono (t/0.05 } \\
\text { ha) }\end{array}$ & 0.21 & 0.81 & 1.04 & 0.76 & 7.01 & 1.85 & 3.11 & 0.00 & 0.00 & 14.79 \\
\hline
\end{tabular}

\section{CONCLUSIONES}

Para el ecosistema de bosques naturales del Helobioma Pacífico-Atrato, ubicado en la cuenca media y baja del río Quito, se presenta una biomasa total de 439,72 t/0.05 ha, las diez especies con mayor índice de valor de importancia representan el $74.38 \%$ del total de la biomasa de este ecosistema, siendo O. platyspermum la quinta especie más importante, aportando la mayor cantidad de biomasa con un $12.14 \%$ del total, seguida de $M$. guyanensis. Este comportamiento no es extraño dada la capacidad de acumulación de biomasa de estas especies ampliamente conocidas por los habitantes de la región como proveedoras de madera para aserrío, así como también por la gran cantidad de individuos con $\mathrm{DAP} \geq 10 \mathrm{~cm}$, con que cuentan.

La distribución de biomasa para el ecosistema en las clases diamétricas VIII (80-90 cm) y IX (90$100 \mathrm{~cm}$ ) contienen al $52.25 \%$ del total de biomasa. Para las especies con mayor IVI, se estableció la mayor representación está en la clase diamétrica IV $(40-50 \mathrm{~cm})$, lo que posiblemente está evidenciando un estado de saturación de la capacidad de carga del sitio, es decir que el bosque toma una dinámica de liberación para que las especies suprimidas y co-dominantes puedan surgir y ampliar el rango de biomasa acumulada por él área, lo que resulta conveniente para la reducción de los tiempos de paso (Meza \& Mora 2003).

En los trece modelos evaluados se presentaron dos grandes grupos, los que estiman solamente en función de DAP los cuales estimaron mayor cantidad de biomasa, mientras que los que estimaron en función de DAP y altura proyectaron menor cantidad de biomasa. Es necesario revisar este comportamiento ya que se evidencia que la arquitectura del bosque (relaciones numéricas entre variables dasométricas) incide significativamente en las estimaciones de biomasa, y por tanto se deberá tener especial atención a la hora de extrapolar resultados, ya que esta arquitectura como se sabe está correlacionada con el nivel de intervención, la calidad de sitio, la dinámica de perturbaciones naturales, la distribución de gremios ecológicos, y las relaciones funcionales entre las especies, entre otras variables.

El modelo más ajustado para el ecosistema fue el propuesto por IPCC (2005) y para las especies con mayor IVI fue el modelo de Chave et al. (2005), situación que no sorprende y que por tanto podría adoptarse como guía para la microrregión. Con base en esto puede afirmarse que el carbono almacenado total dentro del área muestreada para el ecosistema de los bosques naturales del Helobioma Pacífico-Atrato del rio Quito, es de 219.86 t/0.05 ha.

Los análisis mostraron que la capacidad de captura del ecosistema es ligeramente inferior al reportado por Arellano \& Vásquez (2012), esta situación es bastante común debido a la variabilidad de agentes que influyen en las características del bosque, en especial su grado de intervención, y el estado de sucesión en que se encuentre. Aun así, se pone de relieve la capacidad de estos bosques para incidir en los procesos de acumulación de carbono atmosférico, y contribución a mitigar los efectos de la variabilidad climática. 


\section{REFERENCIAS}

[1]. Ardil, S. E. (2009). CONSTRUCCIÓN DE UNIDADES DE APRENDIZAJE ADAPTATIVAS SEGÚN EL CONTEXTO Y BASADA EN EL ESTÁNDAR.

[2]. Basu, P. (2010). Biomass Gasification And Pyrolysis Practical Design . Burlington: AP.

[3]. Mendoza, J., Bula, A., Gómez, R., \& Corredor, L. (2012). Análisis Exergético de la Gasificación de Biomasa. Información Tecnológica. Vol. 23, 5.

[4]. Emun, F., Gadalla, M., Majozi, T., \& Boer, D. (2010). Integrated gasification combined cycle (IGCC) process simulation and optimization. Computers and Chemical Engineering, 331-338.

[5]. Quiceno, D., \& Mosquera, M. (2010). Alternativas tecnológicas para el uso de la cascarilla de arroz como combustible (Vol. 18). Santiago de Cali: Tesis de gradoUniversidad Autónoma del Occidente.

[6]. Escalante, H., Orduz, J., Zapata, H., Cardona, M., \& Duarte, M. (2011). Atlas del Potencial Energético de la Biomasa Residual en Colombia (1.ED ed.). Bucaramanga: UIS.

[7]. Brasió, a. (2013). Nonlinear model predictive control of biodiesel productionvia transesterification of used vegetable oils. Elsevier, 71-79.

[8]. Benavides, P. (2012). Optimal control of biodiesel production in a batch reactor. ELSEVIER, 219-226.

[9]. Conexionismo. (2012). Conexionismo. Obtenido de http://www.conexionismo. com/leer_articulo.php?ref=prueba_t_de_ student_para_dos_muestras_relacionadas902ybsd7

[10]. Tala, D. (2014). Prezi. Obtenido de Ciencia de los materiales: Es el área que aparece bajo la porción elástica de la curva esfuerzodeformación, es la energía elástica que un material absorbe o libera durante la aplicación y liberación de la carga aplicada respectivamente

[11]. Diéguez Fernández, I. (2012). Ensayo de compresión. Obtenido de http:// es.slideshare.net/iadiegue/ensayo-decompresin
[12]. Secretaría de Educación. (2015). Currículo 40x40 Currículo para la excelencia académica y la formación integral. Obtenido de http://www.educacionbogota.edu.co/

[13]. Rojo, Á., Garrido, C., Soto, G., Sáinz, M., Fernández, M. C., \& Hernández, D. (2010). Talleres de enriquecimiento extracurricular para alumnos de altas habilidades. Obtenido de http://www.aufop.com/aufop/ uploaded_files/articulos/1268619297.pdf

[14]. Gonzalez, D., Griful, E., Mudarra, P., \& P, S. (2012). Extracurricular learning program for professional skills development in engineering schools. Obtenido de http:// ieeexplore.ieee.org/xpl/articleDetails. jsp?arnumber $=6397357$

[15]. Hierro, N., Galanis, N., Mayol, E., Casany, M. J., \& Alier, M. (2013). Using a pull mechanism for connecting an informal learning collector with external applications. Obtenido de http://dl.acm.org/citation. cfm?id $=2536571$

[16]. González Vargas, J. C. (23 de Mayo de 2016). RunayaySoft: sistema de recomendación de actividades de enriquecimiento en entornos educativos. Obtenido

[17]. Arellano \& Vásquez . (930 de 2012). ESTRUCTURA, BIOMASA AÉREA Y CARBONO ALMACENADO EN LOS BOSQUES DEL SUR Y NOROCCIDENTE DE CÓRDOBA. (O. Rangel-Ch, Ed.) COLOMBIA DIVERSIDAD BIOTICA, 1.

[18]. IDEAM. (2016). LA CIFRA DE DEFORESTACIÓN EN COLOMBIA 2015 REPORTA 124.035 HECTÁREAS AFECTADAS. Bogotá: MINAMBIENTE.

[19]. IPCC. (2011). The IPCC Special Report on Renewable Energy Sources and Climate Change Mitigation. New York: The New School for Social Research, Co-Chair of the IPCC Working Group III "Mitigation of Climate Change".

[20]. Alvarez. (2012). Contribución de los bosques tropicales de montaña en el almacenamiento de carbono en Colombia. Revista de biologia tropical, Vol 63 No 1. 